\title{
Estudio florístico - palinológico en las localidades de Huarán y Cuyo Grande del Valle Sagrado de los Incas (Cusco, Perú).
}

\section{Floristic - palynological study in the locations of Huarán and Cuyo Grande from the Sacred Valley of the Incas (Cusco, Perú).}

\author{
Raúl Yuca-Rivas \\ Sociedad Botánica del Cusco, Facultad de Ciencias, Universidad Nacional de San Antonio Abad del \\ Cusco, Ciudad Universitaria, Av. De la Cultura s/n, Cusco, Perú. Email: \\ rivas_84_zu@hotmail.com
}

\section{Resumen}

El objetivo de este estudio fue determinar la flora palinológica en las localidades de Huarán y Cuyo Grande delValle Sagrado de los Incas y contribuir al conocimiento palinológico en la región Cusco. Se identificaron en total 120 especies fanerogámicas dentro de un área de 1000 metros de radio alrededor de dichas localidades, durante los meses de enero-abril y julio-octubre de 2013. A partir de las flores obtenidas, se extrajo el polen contenido en ellas para luego realizar preparaciones microscópicas acetolizadas. La observación de los caracteres polínicos se ejecutó sobre 25 granos de polen por especie, tomándose medidas de eje polar (P), plano ecuatorial (E) y algunos elementos esculturales, además se recabaron datos del tipo de polen, ornamentación de la exina y descripción del tamaño del grano de polen; la forma se determinó como la relación P/E. Para los datos cuantitativos se utilizaron los estadísticos descriptivos. Asteraceae, Fabaceae, Lamiaceae, Rosaceae y Solanaceae presentaron mayor número de especies. El tipo de grano de polen más frecuente fue el tricolporado (54.17\%), en cuanto a forma, fue el oblato esferoidal $(41.67 \%)$, en patrón de ornamentación de la exina, el más frecuente fue el reticulado (28.33\%) y en cuanto a tamaño, el mediano fue el más recurrente (45.83\%). Estos resultados sugieren que los caracteres analizados tienen importancia taxonómica, permitiendo la clasificación de las especies estudiadas, y que posteriormente pueden ser utilizados en trabajos aplicados sobre palinología.

Palabras clave: Palinología, flora palinológica, carácter polínico, Huarán, Cuyo Grande.

\begin{abstract}
The aim of this work was to determine the palynological flora in the locations of Huarán and Cuyo Grande from the SacredValley of the Incas and contribute to palynological knowledge in the Cusco region. A total of 120 phanerogamic species were identified within an area of 1000 meters radius around Huarán and Cuyo Grande, during January-April and July-October of 2013. From flowers obtained, pollen content was removed in order to carry out microscopic acetolized preparations. The observations of palynological characters were made on 25 pollen grains per species, taking measurements of polar axis (P), equatorial plane (E) and some sculptural elements, also data of pollen type, ornamentation of exine and description of pollen size was obtained; pollen shape was determined as P/E ratio. Descriptive statistics were used for quantitative data. Asteraceae, Fabaceae, Lamiaceae, Rosaceae and Solanaceae were families with the greatest number of species. The most common pollen type was tricolporate $(54.17 \%)$, in terms of form, oblate spheroidal (41.67\%), in ornamentation pattern of exine, reticulate was the most frequent $(28.33$ $\%)$ and in terms of size, the medium was the most recurrent (45.83\%). These results suggest the analyzed characters have a great taxonomic value, allowing the classification of the species considered, and subsequently they can be used in applied palynological studies.
\end{abstract}

Key words: Palynology, palynological flora, pollen character, Huarán, Cuyo Grande. 


\section{Introducción}

Como parte de la botánica, la taxonomía se ocupa de los principios, reglas y características que se usan para clasificar a los vegetales dentro de grupos específicos. La categoría base en la taxonomía vegetal es la especie. Para determinar una especie y ubicarla dentro de un grupo específico, se suelen utilizar características morfológicas y moleculares. Tanto los granos de polen como las esporas, por su gran variabilidad y constancia, representan excelentes caracteres que pueden ser utilizados en taxonomía. De la misma manera, la morfología polínica es verdaderamente reveladora del parentesco y procedencia de los diversos grupos taxonómicos (Walker \& Doyle 1975, Sáenz 1978, Carretero 1989, Hesse et al. 2009).

El polen representa a la flora, y este puede ser utilizado con diferentes objetivos y en diversas disciplinas, como por ejemplo, en la determinación de mecanismos de polinización, rutas de migración, fuentes de alimento para insectos y otros polinizadores, tipos de mieles, paleobotánica y paleoclimatología, aerobiología y más recientemente en investigaciones forenses (Sáenz 1978, Jones \& Jones 2001, Andrada 2008, Traverse 2007, Hesse et al. 2009).

Existen muchas razones por que el polen es utilizado en este tipo de investigaciones. Primero, los granos de polen son distintivos, fácilmente reconocibles e identificables a nivel de familia, género e incluso especie. Segundo, los granos de polen están rodeados por una capa de esporopolenina, la cual es bastante resistente y no se degrada fácilmente. Tercero, con la identificación de los granos de polen, el origen geográfico de las plantas de las cuales provienen dichos granos, puede ser- -determinado (Sáenz 1978, Jones \& Jones 2001).

Estudios sobre morfología polínica, se han realizado en diversas partes del mundo (Wodehouse 1935, Erdtman 1943, Crisci 1974, Walker \& Doyle 1975, Sáenz 1978, Lin et al. 2005, Hesse et al. 2009), en Latinoamérica (Salgado-Labouriau 1982, Gamerro 1985, Ortiz \& Cogua 1989, Tellería et al. 2003, Tellería 2008) y en el Perú (Isayama 19871988, Bonino \& Paucarmayta 2002, Ventura \& Huamán 2008, Tito \& Vargas 2010).

El objetivo de este estudio, fue determinar la flora palinológica en las localidades de Huarán y Cuyo Grande delValle Sagrado de los Incas en base a la morfología polínica, y así contribuir al conocimiento palinológico en la región Cusco, constituyendo esta información base para otras disciplinas como se mencionó líneas arriba.

\section{Material y métodos}

\section{Características del área de estudio}

Este estudio se realizó en las localidades de Huarán y Cuyo Grande ubicadas en el Valle Sagrado de los Incas, correspondientes a la provincia Calca y región Cusco. Huarán se halla a 59 kilómetros de la ciudad del Cusco, entre las coordenadas UTM $18 \mathrm{~S} 0821575 \mathrm{E}$ $8527152 \mathrm{~N}$ y $0820635 \mathrm{E} 8525713 \mathrm{~N}$ y con altitudes que oscilan entre los 2800 y $3000 \mathrm{~m}$. Cuyo Grande está ubicada a 44 kilómetros de la ciudad del Cusco, entre las coordenadas UTM 19S 0196330E $8517562 \mathrm{~N}$ y $0197020 \mathrm{E}$ $8517610 \mathrm{~N}$ y con altitudes que oscilan entre los 3200 y 4200 m (figura 1). De acuerdo al Mapa Ecológico del Perú, Huarán corresponde al bosque seco Montano Bajo Subtropical (bsMBS) y Cuyo Grande al bosque húmedo Montano Subtropical (bh-MS) (Pérez 2009). 


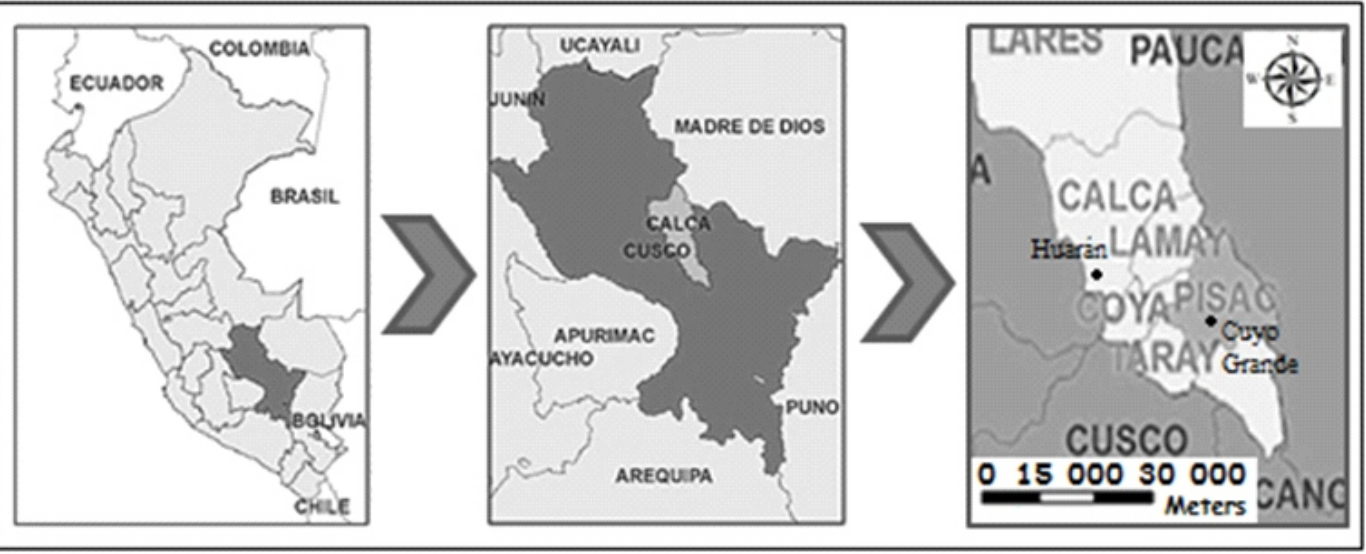

Figura 1: Mapa de ubicación geográfica y política de las localidades de Huarán y Cuyo Grande.

\section{Procedimiento de muestreo}

Se colectaron especies vegetales en floración ubicadas en un área de $1000 \mathrm{~m}$ de radio alrededor de Huarán y Cuyo Grande durante los meses de enero - abril y julio octubre de 2103; adicionalmente, se colectaron flores en antesis de cada planta, estas fueron colocadas en bolsas de papel debidamente rotuladas para su posterior tratamiento en laboratorio. La determinación de las especies se realizó en el Herbario Vargas CUZ de la Facultad de Ciencias de la Universidad Nacional de San Antonio Abad del Cusco (UNSAAC). En lo referente al sistema de clasificación, se consideró los cambios sugeridos por el APG III (2009).

\section{Procesamiento del polen}

Las anteras de las flores fueron procesadas por el método de acetólisis (Erdtman 1960), posteriormente se realizó el montaje de las muestras de polen en gelatina glicerinada para su subsiguiente observación y medición en un microscopio óptico LEICA modelo CME, con micrómetro ocular LEICA (10/100) y su-
- respectivo calibrador que produjo un factor de corrección de 1.10, todas las observaciones se realizaron a 1000 aumentos. El material preparado fue depositado en el Herbario Vargas CUZ.

Para la observación y medición de los caracteres polínicos se utilizaron 25 granos de polen por especie, y se tomaron medidas de eje polar (P), plano ecuatorial (E) y algunos elementos esculturales (espinas, báculos, etc.). También se tomaron datos del tipo de polen, patrón de ornamentación de la exina y descripción del tamaño del grano de polen. La forma de los granos se determinó como la relación $\mathrm{P} / \mathrm{E}$. La terminología usada fue la propuesta por Erdtman (1972) y Hesse et al. (2009).

\section{Análisis estadístico}

Para los datos cuantitativos (caracteres polínicos medidos por especie), se utilizaron estadísticos descriptivos; es decir, el promedio aritmético y la desviación estándar. 


\section{Resultados}

Se determinó 120 especies agrupadas en 105 géneros y 53 familias, siendo Asteraceae, Fabaceae, Lamiaceae, Rosaceae y Solanaceae las familias que presentaron mayor número de especies. Así mismo, se reportaron 91 especies nativas (75.83\%) y 29 introducidas (24.17\%); por otra parte, de las 120 especies, 73 (60.83 $\%)$ correspondieron a Cuyo Grande y 101 $(84.17 \%)$ a Huarán, cabe resaltar que 66 especies (55 \%) fueron comunes a ambas localidades. Sólo en dos especímenes se hizo la determinación a nivel de género, Caiophora sp y Fuchsiasp.

La tabla 1 muestra los datos obtenidos de la caracterización morfológica de los granos de polen de las especies estudiadas, en ella se presenta la media aritmética de la serie de mediciones realizadas junto a la desviación estándar para determinados caracteres polínicos. En algunos casos aparece entre paréntesis una segunda media y desviación estándar, esta información corresponde a aquellos granos de polen que presentaron simetría bilateral en vista polar; es decir, el plano o diámetro ecuatorial no fue uniforme, tomándose dos mediciones por grano de polen para este carácter, un diámetro mayor y uno menor. Por tanto, para estos mismos casos no fue posible determinar la relación $\mathrm{P} / \mathrm{E}$, y la descripción de la forma del grano de polen, se hizo sobre su contorno en vista polar.

De manera similar, en el caso de Lonicera japonica Thunb., Pernettya prostrata (Cav.) DC, Fuchsia sp, Oenothera rosea L'Hér. Ex Aiton y $O$. scabra Krause debido a su morfología polínica, no fue factible realizar mediciones sobre el eje polar. En el caso de algunos elementos esculturales- (por ejemplo espinas y báculos) también se incluye la media aritmética y desviación estándar de las medidas de longitud- -de dichos elementos entre paréntesis, al determinar el patrón de ornamentación de la exina.

El tipo de grano de polen más frecuente fue el tricolporado (54.17\%), en cuanto a forma, fue el oblato esferoidal (41.67\%), en patrón de ornamentación de la exina, el más frecuente fue el reticulado $(28.33 \%)$ y en cuanto a tamaño, el mediano fue el más recurrente $(45.83 \%)$.

\section{Discusión}

Las familias que presentaron mayor número de especies fueron Asteraceae, Fabaceae, Lamiaceae, Rosaceae y Solanaceae, lo que concuerda con lo referido por otros autores sobre la flora en los andes peruanos, siendo efectivamente Asteraceae la predominante (Brako \& Zarucchi 1993, Tupayachi 2011). Es importante referir que existieron muchas especies que fueron comunes para ambas localidades, pero que su estructura poblacional, especialmente en cuanto al número de individuos, fue dispar, ello en mérito a que cada localidad tiene sus particularidades geográficas, biológicas y climatológicas; así como también diferente actividad antrópica, siendo una buena parte de estas especies consideradas con gran potencial económico.

Definir el número de granos de polen que deben ser considerados, especialmente en cuestión de medidas, es término de discusión y debate; en este trabajo se optó por usar 25 granos de polen, así como lo sugiere por ejemplo Sáenz (1978), en contraste a los 10 o 15 granos que se usaron en otras investigaciones (Ubiergo et al. 2009). Por otra parte, algunos autores sugieren que cuanto más grande el número de granos de polen usados- 


\begin{tabular}{|c|c|c|c|c|c|c|c|c|}
\hline \multirow[b]{2}{*}{ FAMILIA } & \multirow[b]{2}{*}{ ESPECIE } & \multicolumn{7}{|c|}{ Caracteres polínicos } \\
\hline & & Eje polar $(\mu \mathrm{m})$ & $\begin{array}{l}\text { Plano ecuatorial } \\
\qquad(\mu \mathrm{m})\end{array}$ & $\mathbf{P} / \mathbf{E}$ & Tipo de polen & Forma del polen & $\begin{array}{c}\text { Ornamentación de la } \\
\text { exina }\end{array}$ & Tamaño \\
\hline \multirow[t]{4}{*}{ ALSTROEMERIACEAE } & Alstroemeria aurea Graham ? н & $42.98 \pm 4.88$ & $\begin{array}{c}99.47 \pm 7.93 \\
(55.3 \pm 6.40)^{*}\end{array}$ & - & Sulcado & Oblongo & Escábrida & Grande \\
\hline & Bomarea dulcis (Hook.) Beauverd ? cG & $37.37 \pm 3.35$ & $\begin{array}{c}65.72 \pm 3.73 \\
(-) *\end{array}$ & & Sulcado & Oblongo & Reticulada & Grande \\
\hline & Bomarea formosissima (Ruiz \& Pav.) Herb. ? " & $38.12 \pm 2.03$ & $\begin{array}{c}68.85 \pm 4.27 \\
(40.99 \pm 2.23)^{*}\end{array}$ & - & Sulcado & Oblongo & Reticulada & Grande \\
\hline & Bomarea ovata (Cav.) Mirb. ? н.CG & $34.67 \pm 1.64$ & $\begin{array}{c}63.07 \pm 2.42 \\
(34.56 \pm 2.17)^{*}\end{array}$ & & Sulcado & Oblongo & Reticulada & Grande \\
\hline AMARYLLIDACEAE & Nothoscordum andicola Kunth? н.сс & $20.84 \pm 0.79$ & $\begin{array}{c}30.83 \pm 1.68 \\
(24.25 \pm 1.06)^{*}\end{array}$ & - & Sulcado & Elíptico & Microrreticulada & Mediano \\
\hline \multirow[t]{2}{*}{ COMMELINACEAE } & Commelina fasciculata Ruiz \& Pav. ?H & $34.51 \pm 2.27$ & $\begin{array}{c}55.08 \pm 2.58 \\
-)^{*}\end{array}$ & & Sulcado & Elíptico & Equinada & Mediano \\
\hline & Setcreasea purpurea B. K. Boom " & $13.88 \pm 0.99$ & $\begin{array}{c}27.43 \pm 1.39 \\
(25.81 \pm 2.50)^{*}\end{array}$ & & Sulcado & Elíptico & Escábrida & Mediano \\
\hline POACEAE & Zea mays L. ${ }^{\text {H.cG }}$ & $72.74 \pm 3.90$ & $66.91 \pm 3.51$ & 1.09 & Ulcerado & Esferoidal & Granulada & Grande \\
\hline XANTHORRHOEACEAE & Kniphofia caulescens Baker ${ }^{\text {H.CG }}$ & $25.27 \pm 1.58$ & $\begin{array}{c}49.36 \pm 2.67 \\
(40.01 \pm 3.95)^{*}\end{array}$ & & Sulcado & Elíptico & Foveolada a reticulada & Mediano \\
\hline AMARANTHACEAE & Amaranthus powellii S. Watson ? H.CG & $25.04 \pm 1.37$ & $26.4 \pm 1.36$ & 0.95 & Pantoporado & Esferoidal & Escábrida & Mediano \\
\hline ANACARDIACEAE & Schinus molle L. ? н.CG & $21.17 \pm 1.89$ & $21.38 \pm 1.54$ & 0.99 & Tricolporado & Oblato esferoidal & Estriada, reticulada & Pequeño \\
\hline APIACEAE & Conium maculatum $\mathrm{L}$. ${ }^{\mathrm{H}} \cdot \mathrm{CG}$ & $25.63 \pm 0.84$ & $13 \pm 0.50$ & 1.97 & Tricolporado & Euprolato & Rugulada, perforada & Pequeño \\
\hline \multirow[t]{23}{*}{ ASTERACEAE } & Achyrocline alata (Kunth) DC. ? CG & $20.97 \pm 0.93$ & $21.01 \pm 1.04$ & 0.99 & Tricolporado & Oblato esferoidal & $\begin{array}{c}\text { Equinada }(2.33 \pm 0.24) \\
\text { perforada }\end{array}$ & Pequeño \\
\hline & Ageratina pentlandiana (DC.) R. King \& H. Rob ? H.cG & $20.52 \pm 1.10$ & $21.01 \pm 1.17$ & 0.98 & Tricolporado & Oblato esferoidal & $\begin{array}{c}\text { Equinada }(4.21 \pm 0.45) \\
\text { perforada }\end{array}$ & Pequeño \\
\hline & Ageratina sternbergiana (DC.) R. King \& H. Rob ? H.CG & $17.09 \pm 0.55$ & $17.64 \pm 0.63$ & 0.97 & Tricolporado & Oblato esferoidal & $\begin{array}{c}\text { Equinada }(3.01 \pm 0.32) \\
\text { perforada }\end{array}$ & Pequeño \\
\hline & Baccharis buxifolia (Lam.) Pers. ? cG & $16.76 \pm 0.71$ & $16.65 \pm 0.58$ & 1.01 & Tricolporado & Esferoidal & $\begin{array}{c}\text { Equinada }(3.06 \pm 0.32) \\
\text { perforada }\end{array}$ & Pequeño \\
\hline & Baccharis Iatifolia (Ruiz \& Pav.) Pers. ? H.cci & $18.15 \pm 0.65$ & $18.92 \pm 0.74$ & 0.96 & Tricolporado & Oblato esferoidal & $\begin{array}{c}\text { Equinada }(3.21 \pm 0.21) \\
\text { perforada }\end{array}$ & Pequeño \\
\hline & Barnadesia horrida Muschl. ? H.cG & $31.7 \pm 2.67$ & $32.13 \pm 2.20$ & 0.99 & Tricolporado & Oblato esferoidal & Lofada & Mediano \\
\hline & Bidens andicola Kunth ? CG & $18.59 \pm 0.61$ & $18.85 \pm 0.70$ & 0.99 & Tricolporado & Oblato esferoidal & $\begin{array}{c}\text { Equinada }(5.21 \pm 0.28) \\
\text { perforada }\end{array}$ & Pequeño \\
\hline & Bidens pilosa L. ? н.cG & $21.16 \pm 0.10$ & $21.36 \pm 1.20$ & 0.99 & Tricolporado & Oblato esferoidal & $\begin{array}{c}\text { Equinada }(4.99 \pm 0.45) \\
\text { perforada }\end{array}$ & Pequeño \\
\hline & Dasyphyllum leiocephalum (Wedd .). Cabrera? H & $34.34 \pm 1.70$ & $29.97 \pm 1,65$ & 1.14 & Tricolporado & Prolato esferoidal & Microequinada & Mediano \\
\hline & Grindelia boliviana Rusby ${ }^{?} \mathrm{CG}$ & $19.73 \pm 0.58$ & $20.06 \pm 0.64$ & 0.98 & Tricolporado & Oblato esferoidal & $\begin{array}{c}\text { Equinada }(3.19 \pm 0.22) \\
\text { perforada }\end{array}$ & Pequeño \\
\hline & Mutisia acuminata (Ruiz \& Pav.), Cabrera? cG & $83.59 \pm 1.98$ & $63.56 \pm 2.13$ & 1.31 & Tricolporado & Subprolato & Verrugosa & Grande \\
\hline & Ophryosporus peruvianus (Gmel.) King \& Rob, ? н & $17.31 \pm 0.57$ & $19.01 \pm 0.57$ & 0.91 & Tricolporado & Oblato esferoidal & Microequinada & Pequeño \\
\hline & Senecio calcensis Cabrera \& Zardini ? CG & $22.77 \pm 0.92$ & $21.49 \pm 0.84$ & 1.06 & Tricolporado & Prolato esferoidal & $\begin{array}{c}\text { Equinada }(3.17 \pm 0.24) \\
\text { perforada }\end{array}$ & Pequeño \\
\hline & Senecio herrerae Cabrera ${ }^{? \mathrm{CG}}$ & $22.63 \pm 1.31$ & $21.76 \pm 1.76$ & 1.04 & Tricolporado & Prolato esferoidal & $\begin{array}{c}\text { Equinada }(3.37 \pm 0.19) \\
\text { perforada }\end{array}$ & Pequeño \\
\hline & Senecio rudbeckiaefolius Meyen \& Walp. ? H.CG & $23,96 \pm 1.27$ & $23.83 \pm 0.96$ & 1.01 & Tricolporado & Esferoidal & $\begin{array}{c}\text { Equinada }(4.27 \pm 0.29) \\
\text { perforada }\end{array}$ & Mediano \\
\hline & Sigesbeckia jorullensis Kunth ${ }^{\text {H }}$ & $23.39 \pm 0.90$ & $24.46 \pm 0.98$ & 0.96 & Tricolporado & Oblato esferoidal & $\begin{array}{c}\text { Equinada }(5.68 \pm 0.34) \\
\text { perforada }\end{array}$ & Mediano \\
\hline & Sonchus asper (L.) Hill ${ }^{\text {H.CG }}$ & $21.76 \pm 0.98$ & $22.68 \pm 1.46$ & 0.96 & Tricolporado & Oblato esferoidal & Equinolofada & Pequeño \\
\hline & Stevia cuzcoensis Hieron. ?H.CGi & $25.59 \pm 1.05$ & $23.58 \pm 0.86$ & 1.08 & Tricolporado & Prolato esferoidal & $\begin{array}{c}\text { Equinada }(3.41 \pm 0.22) \\
\text { perforada }\end{array}$ & Mediano \\
\hline & Tagetes ternif lora kunth? H.CG & $28.75 \pm 1.07$ & $30.6 \pm 1.63$ & 0.94 & Tricolporado & Oblato esferoidal & $\begin{array}{l}\text { Equinada (6.56 } \pm 0.39) \\
\text { perforada }\end{array}$ & Mediano \\
\hline & Taraxacum czuense Schischk. ${ }^{\text {H.CG }}$ & $23.32 \pm 3.25$ & $23.94 \pm 3.51$ & 0.97 & Tricolporado & Oblato esferoidal & Equinolofada & Pequeño \\
\hline & Trixis divari cata (Kunth) Spreng. ? н & $38.93 \pm 1.21$ & $27.59 \pm 0.88$ & 1.41 & Tricolporado & Euprolato & Verrugosa & Mediano \\
\hline & Verbesina auriculigera S. F. Blake? H & $20.53 \pm 1.01$ & $21.25 \pm 1.29$ & 0.97 & Tricolporado & Oblato esferoidal & $\begin{array}{c}\text { Equinada }(5.04 \pm 0.47) \\
\text { perforada }\end{array}$ & Mediano \\
\hline & Viguiera procumbens (Pers.) S. F. Blake? н.сG & $23.72 \pm 0.64$ & $25.61 \pm 1.03$ & 0.93 & Tricolporado & Oblato esferoidal & $\begin{array}{l}\text { Equinada }(6.64 \pm 0.35) \\
\text { perforada }\end{array}$ & Mediano \\
\hline BERBERIDACEAE & Berberis boliviana Lechl. ? H.CG & $45.41 \pm 2.58$ & $44.17 \pm 2.04$ & 1,03 & Espiraperturado & Prolato esferoidal & Escábrida, perforada & Mediano \\
\hline BETULACEAE & Alnus acuminata Kunth ? н-CG & $19.69 \pm 1.28$ & $22.37 \pm 1.49$ & 0.88 & Estefanoporado & Suboblato & Psilada & Pequeño \\
\hline \multirow[t]{2}{*}{ BIGNONIACEAE } & Podranea ricasoliana (Tanfani) Sprague ${ }^{\mathrm{H}}$ & $27 \pm 1.17$ & $36.02 \pm 2.58$ & 0.75 & Tricolpado & Suboblato & Reticulada & Mediano \\
\hline & Tecoma stans (Kunth) J.R.I. Wood ? н & $39.53 \pm 1.38$ & $36.88 \pm 2.01$ & 1.07 & Tricolpado & Prolato esferoidal & Microrreticulada & Mediano \\
\hline \multirow[t]{2}{*}{ BRASSICACEAE } & Raphanus raphanistrum L. ${ }^{H . C G}$ & $21.87 \pm 0.55$ & $22.68 \pm 0.95$ & 0.96 & Tricolpado & Oblato esferoidal & Reticulada & Pequeño \\
\hline & Rapistrum rugosum (L.) All. ${ }^{\mathrm{H}-\mathrm{CG}}$ & $26.51 \pm 1.60$ & $25.06 \pm 1.04$ & 1.06 & Tricolpado & Prolato esferoidal & Reticulada & Mediano \\
\hline \multirow[t]{4}{*}{ CACTACEAE } & Austrocylindropuntia subulata (Muehl.) Backeb. ? H.CG & $82.3 \pm 4.40$ & $84.94 \pm 4.83$ & 0.97 & Pantoporado & Oblato esferoidal & Atectada & Grande \\
\hline & Corryocactus erectus (Backeb.) F. Ritter ? H & $49.46 \pm 2.30$ & $52.92 \pm 2.86$ & 0.93 & Tricolpado & Oblato esferoidal & Escábrida & Mediano \\
\hline & Echinopsis maximiliana Heyder ex A. Dietr, ? H.Ca & $53.68 \pm 3.07$ & $54.16 \pm 2.79$ & 0.99 & Tricolpado & Oblato esferoidal & Microequinada & Grande \\
\hline & Opuntia ficus-indica (L.) Mill. H.CG & $100.17 \pm 4.09$ & $105.68 \pm 4.62$ & 0.95 & Pantoporado & Oblato esferoidal & Reticulada & Muy grande \\
\hline CALCEOLARIACEAE & Calceolaria tripartita Ruiz \& Pav, ?H & $15.97 \pm 0.68$ & $17.05 \pm 0.61$ & 0.94 & Tricolporado & Oblato esferoidal & Microrreticulada & Pequeño \\
\hline CAPRIFOLIACEAE & Lonicera japonica Thunb, ${ }^{\mathrm{H}}$ & - & $75.01 \pm 6.82$ & & Tricolpado & Oblato esferoidal & Equinada $(1.6 \pm 0.46)$ & Grande \\
\hline \multirow[t]{2}{*}{ CONVOLVULACEAE } & Convolvulus crenatifolius Ruiz \& Pav, ? H.CG & $52.38 \pm 1.61$ & $53.95 \pm 1.85$ & 0.97 & Tricolpado & Oblato esferoidal & Atectada & Grande \\
\hline & Ipomoea dumetorum willd. Ex Roem. \& Schult. ?н.са & $85.43 \pm 3.08$ & $86.08 \pm 3.17$ & 0.99 & Pantoporado & Oblato esferoidal & $\begin{array}{l}\text { Equinolofada }(9.45 \pm \\
0.87)\end{array}$ & Grande \\
\hline \multirow[t]{2}{*}{ CUCURBITACEAE } & Cucurbita ficifolia Bouché ${ }^{?} \mathrm{H} \cdot \mathrm{CG}$ & $133.87 \pm 13.89$ & & & Pantoporado & Esferoidal & Equinada $(5.62 \pm 0.75)$ & Muy grande \\
\hline & Sicyos baderoa Hook. \& Arn. ? H.cci & $47.63 \pm 2.95$ & $62.48 \pm 5.04$ & 0.76 & Estefanocolpado & Suboblato & Equinada $(4.64 \pm 0.68)$ & Grande \\
\hline CUNONIACEAE & Weinmannia producta Moricand ${ }^{?} \mathrm{H}$ & $12.94 \pm 0.98$ & $11.24 \pm 0.73$ & 1.15 & Tricolporado & Subprolato & Microrreticulada & Pequeño \\
\hline ELAEOCARPACEAE & Vallea stipularis L. f. ? H & $10.67 \pm 0.55$ & $10.34 \pm 0.65$ & 1.03 & Tricolporado & Prolato esferoidal & Psilada & Muy pequeño \\
\hline
\end{tabular}




\begin{tabular}{|c|c|c|c|c|c|c|c|c|}
\hline ERICACEAE & Pernettya prostrata (Cav.) DC. ? $\mathrm{CG}$ & & $27.94 \pm 0.92$ & & Tricolporado & Oblato esferoidal & Escábrida & Mediano \\
\hline \multirow[t]{2}{*}{ ESCALLONIACEAE } & Escallonia myrtilloides L. f. ? cG & $16.81 \pm 0.75$ & $16.87 \pm 0.63$ & 0.99 & Tricolporado & Oblato esferoidal & Escábrida & Pequeño \\
\hline & Escallonia resinosa (Ruiz \& Pav.) Pers. ? H.CG & $18.44 \pm 0.97$ & $17.89 \pm 0.76$ & 1.03 & Tricolporado & Prolato esferoidal & Psilada & Pequeño \\
\hline EUPHORBIACEAE & Ricinus communis L. ${ }^{H}$ & $32.29 \pm 1.34$ & $32.4 \pm 1.10$ & 0.99 & Tricolporado & Oblato esferoidal & Microrreticulada & Mediano \\
\hline \multirow[t]{6}{*}{ FABACEAE } & Astragalus garbancillo $\mathrm{Cav} . ? \mathrm{CG}$ & $26.49 \pm 0.85$ & $18.94 \pm 0.69$ & 1.39 & Tricolporado & Euprolato & Foveolada a reticulada & Pequeño \\
\hline & Caesalpinia spinosa (Feuillée ex Molina) Kuntze ? н & $41.42 \pm 2.19$ & $47.2 \pm 1.43$ & 0.88 & Tricolporado & Oblato esferoidal & Reticulada & Mediano \\
\hline & Medicago polymorpha L. ${ }^{\text {H.ca }}$ & $19.71 \pm 1.01$ & $15.88 \pm 0.68$ & 1.24 & Tricolporado & Subprolato & Perforada a foveolada & Pequeño \\
\hline & Senna versicolor (Vogel) Irwin \& Barneby? н.СG & $26.41 \pm 1.41$ & $19.17 \pm 0.87$ & 1.38 & Tricolporado & Euprolato & Escábrida & Pequeño \\
\hline & Spartium junceum L. ${ }^{\text {H.CG }}$ & $39.73 \pm 1.50$ & $38.63 \pm 2.01$ & 1.03 & Tricolporado & Prolato esferoidal & Foveolada & Mediano \\
\hline & Trifolium repens L. H.CG & $24.42 \pm 2.45$ & $19.49 \pm 2.13$ & 1.25 & Tricolporado & Subprolato & Foveolada a reticulada & Pequeño \\
\hline GENTIANACEAE & Gentianella persquarrosa (Reimers) J. S. Pringle ? CG & $33.1 \pm 1.30$ & $35.26 \pm 1.52$ & 0.94 & Tricolporado & Oblato esferoidal & Reticulada & Mediano \\
\hline GERANIACEAE & Geranium herrerae Knuth ? H.CG & $55.46 \pm 3,09$ & $61.83 \pm 2.55$ & 0.89 & Tricolporado & Oblato esferoidal & $\begin{array}{l}\text { Reticulada, clavada } \\
\text { (reticulum cristatum) }\end{array}$ & Grande \\
\hline \multirow[t]{5}{*}{ LAMIACEAE } & Leonotis nepetifolia (L.)R. Br. " & $43.16 \pm 1.44$ & $27.43 \pm 1.82$ & 1.57 & Tricolpado & Prolato esferoidal & Reticulada & Mediano \\
\hline & Lepechinia floribunda (Benth.) Epling? H & $31.81 \pm 1.11$ & $\begin{array}{c}44.06 \pm 1.65 \\
(38.39 \pm 1.35)^{*}\end{array}$ & & Estefanocolpado & Elíptico & Reticulada & Mediano \\
\hline & Minthostachys acris Schmidt-Leb. ? H.CG & $24.31 \pm 1.40$ & $\begin{array}{l}27.67 \pm 1.28 \\
(25.09 \pm 1.80)^{*}\end{array}$ & & Estefanocolpado & Elíptico & Reticulada & Mediano \\
\hline & Rosmarinus officinalis $\mathrm{L} .{ }^{\mathrm{H}}$ & $39.58 \pm 2.49$ & $46.55 \pm 2.84$ & 0.85 & Estefanocolpado & Suboblato & Reticulada & Mediano \\
\hline & Salvia oppositiflora Ruiz \& Pav. ?cG & $32.94 \pm 1.35$ & $\begin{array}{c}44.82 \pm 2.97 \\
(34.78 \pm 3.15)^{*}\end{array}$ & - & Estefanocolpado & Elíptico & Birreticulada & Mediano \\
\hline LOASACEAE & Caiophora sp ? н & $23.41 \pm 1.14$ & $18.66 \pm 0.76$ & 1.25 & Tricolporado & Subprolato & Escábrida & Pequeño \\
\hline LORANTHACEAE & Gaiadendron punctatum (Ruiz \& Pav.) G. Don ?H & $11.15 \pm 0.64$ & $21.12 \pm 1.26$ & 0.53 & Sincolpado & Triangular cóncavo & Escábrida & Pequeño \\
\hline MALVA CEAE & Alcea rosea L. ${ }^{\text {H.cG }}$ & $105.73 \pm 3.81$ & $106.16 \pm 3.10$ & 0.99 & Pantoporado & Oblato esferoidal & Equinada $(11.18 \pm 1.01)$ & Muy grande \\
\hline MYRICACEAE & Morella pubescens (Humb \& Bonpl Ex Willd) Wilb. ? н & $22.03 \pm 1.01$ & $23.76 \pm 1.35$ & 0.93 & Triporado & Oblato esferoidal & Psilada & Pequeño \\
\hline \multirow[t]{2}{*}{ MYRTACEAE } & Eucalyptus globulus Labill, н.с-с & $17.29 \pm 1.33$ & $27.41 \pm 1.68$ & 0.63 & Sincolporado & Euoblato & Psilada & Pequeño \\
\hline & Luma chequen (Feuillée ex Molina) A. Gray H.CG & $14.37 \pm 0.92$ & $19.87 \pm 0.98$ & 0.72 & Sincolporado & Euoblato & Escábrida & Pequeño \\
\hline NYCTAGINACEAE & Bougainvillea spectabilis Willd. ${ }^{\text {H }}$ & $42.23 \pm 4.11$ & $43.36 \pm 5.08$ & 0.97 & Tricolpado & Oblato esferoidal & Reticulada & Mediano \\
\hline \multirow[t]{4}{*}{ ONAGRACEAE } & Fuchsia boliviana Carriére? н & $46.11 \pm 3.81$ & $58,08 \pm 4.53$ & 0.79 & Diporado & Suboblato & Escábrida & Grande \\
\hline & Fuchsia sp & & $70.31 \pm 5.70$ & & Triporado & Suboblato & Granulada & Grande \\
\hline & Oenothera rosea L'Hér. Ex Aiton ? н.CG & & $47.2 \pm 2.19$ & - & Triporado & Oblato esferoidal & Granulada & Grande \\
\hline & Oenothera scabra Krause ? CG & & $71.44 \pm 2.13$ & & Triporado & Oblato esferoidal & Granulada & Grande \\
\hline OROBANCHACEAE & Bartsia camporum Weddell ? н.сG & $28.94 \pm 0.96$ & $31.54 \pm 1.02$ & 0.92 & Tricolpado & Oblato esferoidal & Escábrida & Mediano \\
\hline OXALIDACEAE & Oxalis peduncularis Kunth? ${ }^{\text {H }}$ & $27.38 \pm 0.83$ & $25.6 \pm 0.91$ & 1.07 & Tricolpado & Prolato esferoidal & Reticulada & Mediano \\
\hline PAPAVERACEAE & Eschscholzia californica Cham. ${ }^{\text {н.cG }}$ & $28.57 \pm 1.44$ & $37.15 \pm 1.29$ & 0.77 & Estefanocolpado & Suboblato & $\begin{array}{l}\text { Reticulada, microequinada } \\
\text { (reticulum cristatum) }\end{array}$ & Mediano \\
\hline \multirow[t]{2}{*}{ PASSIFLORACEAE } & Passiflora pinnatistipula Cav. ? ${ }^{\mathrm{cG}}$ & $59.67 \pm 2,81$ & $63.23 \pm 2.26$ & 0.94 & Sincolpado & Oblato esferoidal & Reticulada & Grande \\
\hline & Passiffora tripartita (Juss.) Poir. ? н.сCG & $62.86 \pm 3.59$ & $66.37 \pm 2.91$ & 0.95 & Sincolpado & Oblato esferoidal & Reticulada & Grande \\
\hline PHRYMACEAE & Mimulus glabratus Kunth? H.CG & $27.86 \pm 4.85$ & $35.26 \pm 5.90$ & 0.79 & Espiraperturado & Suboblato & Microrreticulada & Mediano \\
\hline PHYTOLACCACEAE & Phytolacca bogotensis Kunth? H & $27.76 \pm 2.34$ & $29.11 \pm 2.94$ & 0.95 & Tricolporado & Oblato esferoidal & Granulada & Mediano \\
\hline PLANTAGINACEAE & Plantago australis Lam. ? н.CG & $23.23 \pm 0.86$ & $24.31 \pm 1.02$ & 0.96 & Pantoporado & Suboblato & Verrugosa & Pequeño \\
\hline POLEMONIACEAE & Cantua buxifolia Juss. Ex Lam. ? CG & $71.3 \pm 3.58$ & $73.35 \pm 3.36$ & 0.97 & Pantoporado & Oblato esferoidal & $\begin{array}{l}\text { Reticulada, gemada } \\
\text { (reticulum cristatum) }\end{array}$ & Grande \\
\hline \multirow[t]{2}{*}{ POLYGONACEAE } & Polygonum hydropiperoides Michx. ? H & $52.38 \pm 3.68$ & $52.76 \pm 3.63$ & 0.99 & Pantoporado & Oblato esferoidal & Reticulada & Grande \\
\hline & Rumex cuneifolius Campd. ? H.CG & $25.87 \pm 1.81$ & $28.35 \pm 1.83$ & 0.91 & Tricolporado & Oblato esferoidal & Perforada a foveolada & Mediano \\
\hline \multirow[t]{2}{*}{ RANUNCULACEAE } & Clematis seemannii kuntze $^{?} \mathrm{cG}$ & $18.26 \pm 1.18$ & $19,01 \pm 1.52$ & 0.96 & Tricolpado & Oblato esferoidal & Microequinada & Pequeño \\
\hline & Ranunculus praemorsus Kunth ex DC. ? н.CG & $34.4 \pm 2.28$ & $36.07 \pm 2.51$ & 0.95 & Pantocolpado & Oblato esferoidal & Microequinada & Mediano \\
\hline RHAMNACEAE & Colletia spinosissima J.F. Gmel. • н.сG & $27.32 \pm 1.84$ & $26.95 \pm 1.13$ & 1.01 & Tricolporado & Prolato esferoidal & Escábrida & Mediano \\
\hline \multirow[t]{6}{*}{ ROSACEAE } & Hesperomeles ferruginea (Pers.) Benth. ${ }^{\bullet}$ & $34.54 \pm 1.81$ & $32.16 \pm 1.84$ & 1.07 & Tricolporado & Prolato esferoidal & Estriada a rugulada & Mediano \\
\hline & Kageneckia lanceolata Ruiz \& Pav. $\bullet$ & $31.43 \pm 2.42$ & $33.21 \pm 2.52$ & 0.95 & Tricolporado & Oblato esferoidal & Estriada & Mediano \\
\hline & Polylepis racemosa Ruiz \& Pav, • н.сс & $24 \pm 1.11$ & $25.06 \pm 1.19$ & 0.96 & Tricolporado & Oblato esferoidal & Verrugosa & Mediano \\
\hline & Prunus persica (L.) Batsch H.cci & $41.9 \pm 2.04$ & $40.5 \pm 2.89$ & 1.03 & Tricolporado & Prolato esferoidal & Estriada & Mediano \\
\hline & Prunus serotina Ehrh. ${ }^{\text {H.cG }}$ & $25.78 \pm 1.38$ & $22.84 \pm 1.24$ & 1.13 & Tricolporado & Prolato esferoidal & Estriada & Pequeño \\
\hline & Rubus urticifolius Poir. $\bullet^{\text {H }}$ & $22.04 \pm 1.94$ & $16.94 \pm 1.19$ & 1.30 & Tricolporado & Subprolato & Estriada & Pequeño \\
\hline RUTACEAE & Ruta chalepensis L. н.CG & $22.57 \pm 1.02$ & $20.99 \pm 0.80$ & 1.07 & Tricolporado & Prolato esferoidal & Estriada & Pequeño \\
\hline \multirow[t]{2}{*}{ SALICACEAE } & Pineda incana Ruiz \& Pav. •н & $21.65 \pm 1.28$ & $18.46 \pm 1.12$ & 1.17 & Tricolporado & Subprolato & Reticulada & Pequeño \\
\hline & Salix humboldtiana Willd. ${ }^{*}$ H.cG & $19.32 \pm 0.96$ & $16.96 \pm 0.77$ & 1.14 & Tricolpado & Subprolato & Reticulada & Pequeño \\
\hline SAPINDACEAE & Serjania squarrosa Radlk. ${ }^{\bullet}$ & $19.6 \pm 0.96$ & $30.02 \pm 1.42$ & 0.65 & Sincolporado & Euoblato & Reticulada, foveolada & Mediano \\
\hline \multirow[t]{2}{*}{ SCROPHULARIACEAE } & Buddleja coriacea Remy ${ }^{\bullet} \mathrm{ca}$ & $27.87 \pm 1,46$ & $23.52 \pm 1.78$ & 1.18 & Tricolporado & Subprolato & Perforada a foveolada & Pequeño \\
\hline & Verbascum virgatum Stokes ${ }^{\mathrm{H}}$ & $26.03 \pm 1.19$ & $28.07 \pm 1.91$ & 0.93 & Tricolporado & Oblato esferoidal & Reticulada & Mediano \\
\hline SOLANACEAE & Brugmansia arborea (L.) Lagerh. $\bullet^{\mathrm{H} . \mathrm{CG}}$ & $34.56 \pm 1.61$ & $40.61 \pm 1.82$ & 0.85 & Tricolporado & Suboblato & Estriada, foveolada & Mediano \\
\hline & Brugmansia sanguinea (Ruiz \& Pav.) D. Don • н.сCG & $35.59 \pm 1.65$ & $39.74 \pm 1.35$ & 0.89 & Tricolporado & Oblato esferoidal & Estriada, foveolada & Mediano \\
\hline & Cestrum conglomeratum Ruiz \& Pav. $\bullet_{\mathrm{H}}$ & $38.39 \pm 2.17$ & $28.51 \pm 1.19$ & 1.35 & Tricolporado & Euprolato & Escábrida & Mediano \\
\hline & Datura stramonium L. ${ }^{H}$ & $39.26 \pm 1.74$ & $42.61 \pm 1.41$ & 0.92 & Tricolporado & Oblato esferoidal & Estriada, reticulada & Mediano \\
\hline & Dunalia oborata (Ruiz \& Pav.) Dammer ${ }^{\bullet \text { н.ca }}$ & $28.86 \pm 0.94$ & $27.5 \pm 0.73$ & 1.05 & Tricolporado & Prolato esferoidal & Escábrida & Mediano \\
\hline & Jaltomata herrerae (C.V. Morton) Mione $\bullet^{\mathrm{CG}}$ & $36.56 \pm 1.60$ & $31.1 \pm 1.32$ & 1.17 & Tricolporado & Subprolato & Escábrida & Mediano \\
\hline & Nicandra physalodes (L.) Gaertn. ${ }^{\bullet}$ & $29.54 \pm 1.57$ & $27.65 \pm 1.70$ & 1.07 & Tricolporado & Prolato esferoidal & Escábrida & Mediano \\
\hline & Nicotiana tomentosa Ruiz \& Pav. $\bullet_{\text {H.cG }}$ & $30.43 \pm 1.28$ & $23.78 \pm 0.77$ & 1.28 & Tricolporado & Subprolato & Estriada, verrugosa & Mediano \\
\hline & Solanum maturecalvans Bitter $\bullet^{\bullet}$ & $20.37 \pm 0.58$ & $17.91 \pm 0.84$ & 1.14 & Tricolporado & Prolato esferoidal & Psilada & Pequeño \\
\hline & Solanum nitidum Ruiz \& Pav. $\bullet^{\mathrm{cG}}$ & $29.35 \pm 1.30$ & $27.57 \pm 1.40$ & 1.06 & Tricolporado & Prolato esferoidal & Escábrida & Mediano \\
\hline TROPAEOLACEAE & Tropaeolum majus L. ${ }^{\text {H.CG }}$ & $27.86 \pm 1.82$ & $32.45 \pm 2.01$ & 0.86 & Tricolpado & Suboblato & Reticulada & Mediano \\
\hline & Tropaeolum peregrinum L. $\bullet$ н.cG & $21.87 \pm 1.56$ & $25.54 \pm 2.02$ & 0.86 & Tricolpado & Suboblato & Reticulada & Pequeño \\
\hline VERBENACEAE & Duranta armata Moldenke ${ }^{\bullet}$ н & $29.21 \pm 1.29$ & $35.26 \pm 1.31$ & 0.83 & Tricolporado & Suboblato & Escábrida & Mediano \\
\hline
\end{tabular}

(*) Media aritmética y desviación estándar del diámetro ecuatorial menor en granos de polen con simetría bilateral. (?) Especies nativas. (H) Especie colectada en Huarán. (CG) Especie colectada en Cuyo Grande. 
-en las mediciones, se logrará una mayor precisión, y por tanto una mejor diferenciación entre especies o géneros (Salgado-Labouriau 1982, Tito \& Vargas 2010). Pero es importante resaltar que un número extremadamente grande de granos de polen medidos, dota de una sobre sensibilidad poco aplicable en casos prácticos de la palinología. En todo caso, la determinación del número de granos de polen a medir, dependerá de la naturaleza de la investigación y de la aplicación adecuada de la estadística.
Conocer la flora palinológica de un área determinada es importante, pues ella nos proporcionará con fidelidad, la identidad de los granos de polen en trabajos de investigación aplicados, como melisopalinología, aeropalinología, paleobotánica, etc., que puedan ejecutarse en dicho área o zonas contiguas; así mismo, el valor de la palinología en la sistemática vegetal es bastante reconocido (Sáenz 1978; Jones \& Jones 2001; Colinvaux et al. 1999; Hesse et al. 2009).

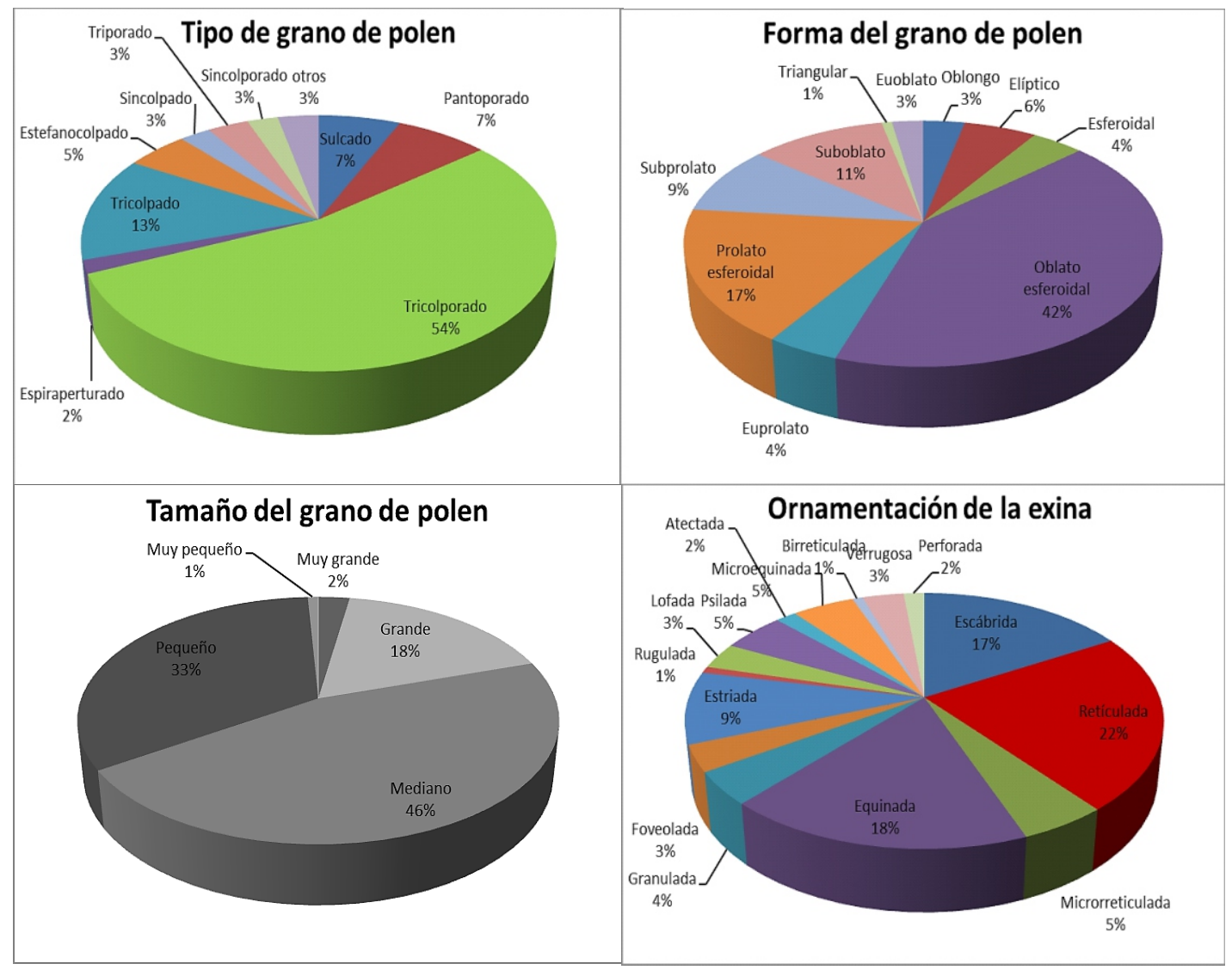

Figura 2: Proporciones (\%) de los principales caracteres polínicos medidos para las 120 especies determinadas en las localidades de Huarán y Cuyo Grande. 
El análisis de los caracteres morfológicos en los granos de polen de las especies estudiadas, muestra que el polen tricolporado es el más recurrente, debido a la predominancia en especies de Asteraceae, esta familia se caracteriza por presentar polen tricolporado, normalmente prolato esferoidal a subprolato y con un patrón de ornamentación de la exina equinado (Wodehouse 1935; Erdtman 1943; Sáenz 1978; Salgado-Labouriau 1982; Carretero 1989; Colinvaux et al. 1999; Hesse et al.2009).

El patrón de ornamentación de la exina presentó diferencias importantes entre las - -especies estudiadas, variando desde el psiladohasta el reticulado, siendo este último el más frecuente. Este carácter contribuye con la separación a nivel específico. SalgadoLabouriau (1982), Lapp \& Castro (2004) y Arias (2005) señalan que los caracteres de la exina fueron relevantes para separar especies dentro de un mismo género.

Estos resultados sugieren que los caracteres analizados, tales como patrón de ornamentación de la exina, tamaño y forma del grano de polen, tienen importancia taxonómica permitiendo la clasificación de las especies estudiadas.
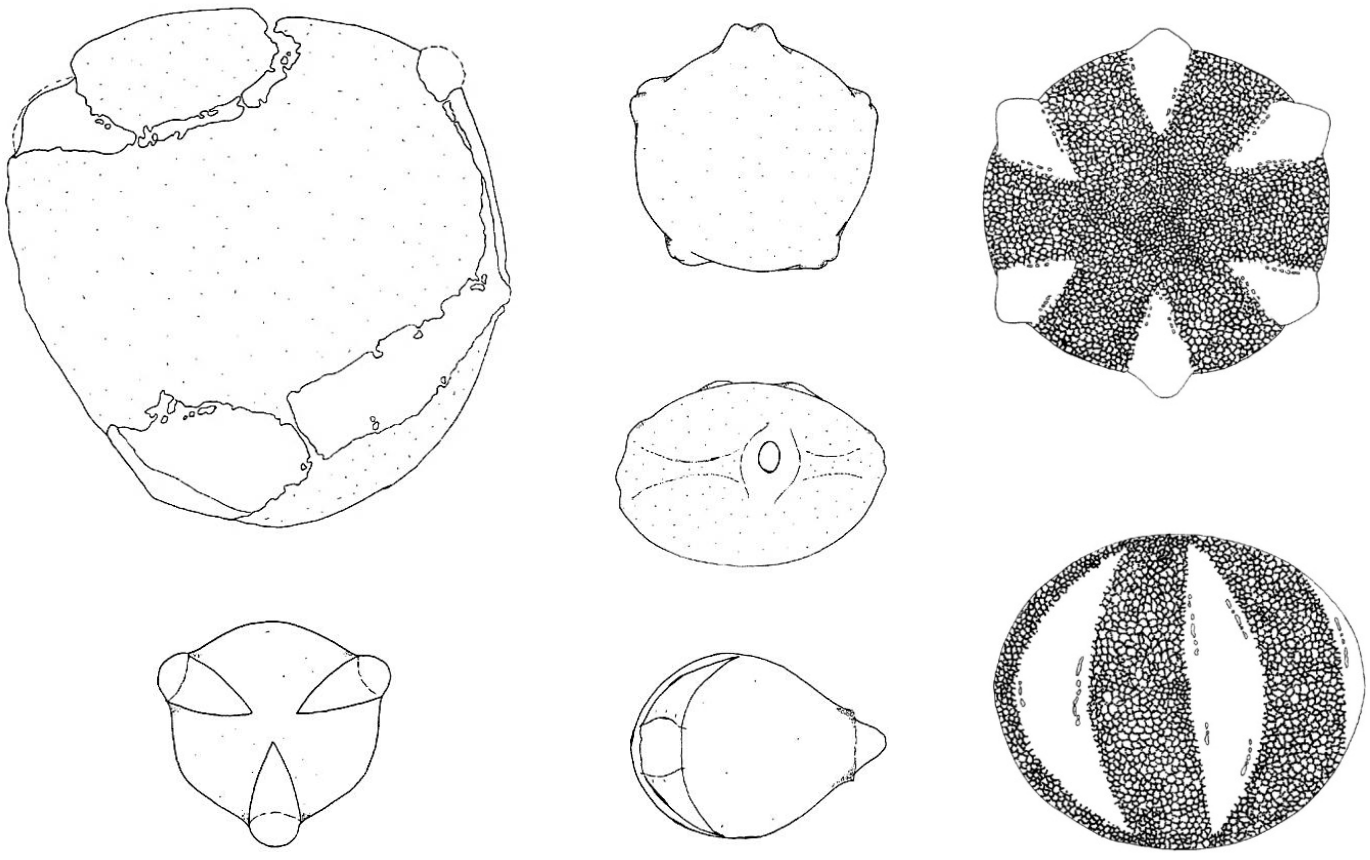

Figura 3: Berberis boliviana Lechl. (A), Alnus acuminata Kunth - vista polar (B), A. acuminata Kunth - vista ecuatorial (C), Minthostachys acris Schmidt-Leb. - vista polar (D), M. acris Schmidt-Leb. - vista ecuatorial (E), Escallonia resinosa (Ruiz \& Pav.) Pers. - vista ecuatorial (F), E. resinosa (Ruiz \& Pav.) Pers. - vista polar (G). 
Estudios sobre morfología polínica en otras partes del Perú y Latinoamérica (SalgadoLabouriau 1982; Isayama 1987-1988; Ortiz \& Cogua 1989; Bonino \& Paucarmayta 2002, Ventura \& Huamán 2008; Tito \& Vargas 2010), ratifican los resultados obtenidos en esta investigación. Familias como Asteraceae, Fabaceae, Rosaceae, Solanaceae, Passifloraceae, Myrtaceae y Lamiaceae, se caracterizan por presentar granos equinados, perforados a reticulados, estriados, escábridos, reticulados, psilados y reticulados respectivamente. En cuanto a tamaño y forma, en estas mismas familias predominan los granos de polen oblato esferoidales a prolato esferoidales y de medianos a grandes.

\section{Agradecimientos}

A la M. Sc. Fructuosa De LaTorre por el acceso al Herbario Vargas CUZ, a la Quim. Alicia Claverí por el acceso al laboratorio de Bioquímica de la Universidad Nacional de San Antonio Abad del Cusco, y finalmente a la Blga. Susana Molleapaza por sus aportes en la revisión del manuscrito preliminar.

\section{Literatura citada}

Andrada, A. C. 2008. La palinología y la miel: origen y tipificación. Agro U.N.S., 5 (10): 5-13.

APG III. 2009. An update of the Angiosperm Phylogeny Group classification for the orders and families of flowering plants. Botanical Journal of the Linnean Society, 162: $105-121$.

Arias, J. 2005. Revisión taxonómica del género Stevia Cav. (Asteraceae-Eupatorieae) en Venezuela. Trabajo de postgrado de Botánica Agrícola. Universidad Central de Venezuela. Maracay.
Bonino, R. E. \& Paucarmayta, D. J. 2002. Evaluación del potencial de la flora melífera en la margen derecha del rio Mapacho, sector Challabamba - Acobamba. PROMANU, Cusco.

Brako, L. \& Zarucchi, J. L. 1993. Catálogo de las Angiospermas y Gimnospermas del Perú. Monographs in systematic botany from the Missouri Botanical Garden. Vol. 45. Missouri Botanical Garden, USA.

Carretero, J. L. 1989. Análisis polínico de la miel. Madrid: Ediciones Mundi - Prensa, España.

Colinvaux, P., De Oliveira, P. E. \& Moreno, J. E. 1999. Amazon Pollen Manual and Atlas. Singapore: Overseas Publishers Association.

Crisci, J.V. 1974. A numerical-taxonomic study of the subtribe Nassauviinae (Compositae, Mutisieae). Journal of the Arnold Arboretum, 55: 568-610.

Erdtman, G. 1943. An Introduction to Pollen Analysis. Maltham, Mass., U.S.A. Published by the Chronica Botanica Company.

1960. The acetolysis method, a revised description. Sven BotTidskr 54: 561 -564 .

1972. Pollen morphology and plant taxonomy, Angiosperms: An introduction to Palynology I. Hafner Publishing Company. NewYork.

Gamerro, J. C. 1985. Morfología del polen de Huarpea y su relación con Barnadesia (Mutisieae, Compositae). Darwiniana, 26(1-4): 43-51.

Hesse, M., Halbritter, H., Zetter, R., Weber, M., Buchner, R., Frosch-Radivo, A. \& Ulrich, S. 2009. Pollen Terminology: An illustrated handbook. Vienna: Springer Wien NewYork, Austria.

Isayama, V. 1987 - 1988. Morfología polínica de algunas especies de la flora del Dpto. de Lambayeque. Zonas Áridas, 5:45-60. 
Jones, G. D. \& Jones, S. D. 2001. The uses of pollen and its implication for entomology. Neotropical Entomology, 30 (3): $341-$ 350 .

Lapp, M. \& Castro, M. 2004. Estudio palinológico de especies venezolanas del género Oyedaeae DC. (Asteraceae). Ernstia 14(1-4): 53-67.

Lin, N.,Wang, H., Li, D. \& Blackmore, S. 2005. Pollen morphology of eight genera of the subtribe Mutisiinae Less. Sensu Bremer (Compositae) from Asia. Journal of Integrative Plant Biology, 47(9): 1036 1046.

Ortiz, D. \& Cogua, J. 1989. Reconocimiento de granos de polen de algunas plantas melíferas en la sabana de Bogotá. Agronomía Colombiana, 6: 52-63.

Pérez, I. M. 2009. Variabilidad morfológica de cultivares de Oxalis tuberosa Mol. (Oca) en tres comunidades campesinas del distrito de Pisac, Calca, Cusco. [Tesis para optar el título profesional de Biólogo]. Universidad Nacional de San Antonio Abad del Cusco.

Sáenz, C. 1978. Polen y Esporas: Introducción a la palinología y vocabulario palinológico. (Primera edición). Madrid: Ediciones H. Blume. España.

Salgado-Labouriau, M. L. 1982. On cavities in spines of Compositae pollen. Grana, 21: 97 $-102$.

Tellería, M. C. 2008. Taxonomy significance of pollen types in the Guayana Highlandcentred composite genera of Mutisioideae (Asteraceae). Botanical Journal of the Linnean Society 156:327-340.

Telleria, M. C., Urtubey, E. \& Katinas, L. 2003. Proustia and Lophopappus (Asteraceae, Mutisieae): Generic and subtribal relationships based on pollen morphology. Review of palaeobotany and palynology, 123:237-246
Tito, J. \& Vargas, N. 2010. Palinotaxonomía y Evolución del género Passiflora L., subgénero Passiflora L. (Passifloraceae) de la región Cusco. [Tesis para optar el título profesional de biólogo]. Universidad Nacional de San Antonio Abad del Cusco.

Tupayachi, A. 2011. Flora fanerogámica del distrito deYucay. Rev. Q'euña, 4: 15-28.

Traverse, A. 2007. Paleopalynology, second edition. Volume 28. Topics in Geobiology. Springer, the Netherlands.

Ubiergo, P., Lapp, M. \& Torrecilla, P. 2009. Morfología del polen de especies de Gongylolepis (Mutisieae: Asteraceae) de la Guayana venezolana. Anales del Jardín Botánico de Madrid. 66(1): 93-107.

Ventura, K. \& Huamán, L. 2008. Morfología polínica de la familia Fabaceae de la parte baja de los valles de Pativilca y Fortaleza (Lima, Perú). Biologist, 6: 112-134.

Walker, J. \& Doyle, J. 1975. The bases of Angiosperm phylogeny: Palynology. Annals of the Missouri Botanical Garden, 62 (3): $480-521$.

Wodehouse, R. P. 1935. Pollen Grains: their structure, identification and significance in science and medicine. McGraw - Hill Book Co. NewYork. 